Supplement of Hydrol. Earth Syst. Sci., 24, 2365-2378, 2020

https://doi.org/10.5194/hess-24-2365-2020-supplement

(c) Author(s) 2020. This work is distributed under

the Creative Commons Attribution 4.0 License.

(c) (1)

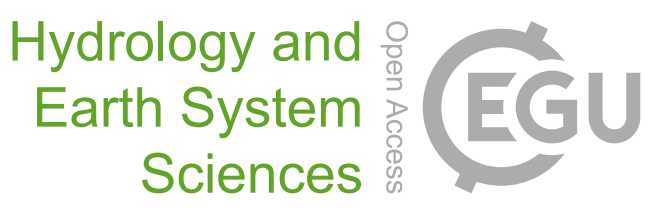

Supplement of

\title{
A line-integral-based method to partition climate and catchment effects on runoff
}

Mingguo Zheng

Correspondence to: Mingguo Zheng (mgzheng@ soil.gd.cn)

The copyright of individual parts of the supplement might differ from the CC BY 4.0 License. 
Table S1 Data of the Batalling Ck

\begin{tabular}{|r|r|r|r|}
\hline \multicolumn{1}{|l|}{ Year } & $R \mathrm{~mm}$ & \multicolumn{1}{l|}{$P \mathrm{~mm}$} & \multicolumn{1}{l|}{$E_{0} \mathrm{~mm}$} \\
\hline 1979 & 8.156159 & 464.4431 & 1089.347 \\
\hline 1980 & 19.64627 & 670.9428 & 1087.463 \\
\hline 1981 & 42.57434 & 687.5864 & 1109.123 \\
\hline 1982 & 53.15154 & 675.1814 & 1066.039 \\
\hline 1983 & 79.30157 & 822.5461 & 1100.647 \\
\hline 1984 & 40.90505 & 610.9478 & 1069.336 \\
\hline 1985 & 46.05864 & 624.9977 & 1113.95 \\
\hline 1986 & 18.24088 & 502.6219 & 1050.854 \\
\hline 1987 & 9.915949 & 467.911 & 1110.182 \\
\hline 1988 & 78.16621 & 771.4137 & 1189.523 \\
\hline 1989 & 36.22554 & 626.2132 & 1078.752 \\
\hline 1990 & 33.37795 & 631.4451 & 1028.017 \\
\hline 1991 & 37.67235 & 688.0311 & 1109.712 \\
\hline 1992 & 41.75197 & 743.0613 & 1009.535 \\
\hline 1993 & 34.55473 & 613.4228 & 1043.555 \\
\hline 1994 & 20.21549 & 472.8909 & 1159.976 \\
\hline 1995 & 23.11371 & 656.5664 & 1125.72 \\
\hline 1996 & 71.81743 & 833.4983 & 1141.496 \\
\hline 1997 & 12.74668 & 505.7044 & 1093.114 \\
\hline 1998 & 27.45874 & 714.279 & 1082.99 \\
\hline 1999 & 24.45006 & 683.1997 & 1105.238 \\
\hline 2000 & 35.67167 & 645.8951 & 1087.463 \\
\hline 2001 & 6.404027 & 451.4155 & 1045.556 \\
\hline 2002 & 24.55285 & 634.5722 & 1095.468 \\
\hline 2003 & 29.86755 & 646.0289 & 1103.002 \\
\hline 2004 & 31.36959 & 646.0731 & 1121.13 \\
\hline 2005 & 41.08764 & 714.5904 & 1036.845 \\
\hline 2006 & 5.214965 & 457.8624 & 1063.802 \\
\hline 2007 & 25.29698 & 716.754 & 1068.393 \\
\hline 2008 & 27.9267 & 641.0637 & 1012.949 \\
\hline & & & \\
\hline
\end{tabular}

\section{Calculation steps}

1) Data preparation and dividing the evaluation period into a number of subperiods Table S2 Data preparation

\begin{tabular}{|c|c|c|c|c|c|c|c|c|c|c|}
\hline Period & Subperiod & $R$ & $P$ & $E_{0}$ & $\begin{array}{c}E= \\
P-R\end{array}$ & $n$ & $\Delta R$ & $\Delta P$ & $\Delta E_{0}$ & $\Delta n$ \\
\hline Reference period & $1979-1984$ & 40.6 & 655 & 1087 & 614 & 3.0 & & & & \\
\hline Subperiod 1 & $1985-1991$ & 37.1 & 616 & 1097 & 579 & 2.9 & -3.5 & -39.2 & 10.3 & -0.19 \\
\hline
\end{tabular}




\begin{tabular}{|l|l|l|l|l|l|l|l|l|l|l|}
\hline Subperiod 2 & $1992-1998$ & 33.1 & 648 & 1094 & 615 & 3.2 & -4.0 & 32.4 & -3.5 & 0.38 \\
\hline Subperiod 3 & $1999-2008$ & 25.2 & 624 & 1074 & 599 & 3.5 & -7.9 & -24.7 & -19.8 & 0.22 \\
\hline
\end{tabular}

2) Calculating $\Delta R_{P}, \Delta R_{E 0}$, and $\Delta R_{n}$ for Subperiod 1

(1) Determining the integral path $L$ :

$L$ is given by parametric equations: $P=\Delta P t+P_{1}, E_{0}=\Delta E_{0} t+E_{01}, \quad n=\Delta n t+n_{1}$, $t \in[0,1]$. Using the data in Table S2, we get:

$$
P=-39.2 t+655, E_{0}=10.3 t+1087, \quad n=-0.19 t+3.0, \quad t \in[0,1]
$$

(2) Calculating $\Delta R_{P}$ using equations as follows:

$$
\begin{aligned}
& \Delta R_{P} \approx 0.001 \Delta P \sum_{i=0}^{999} R_{P}\left(t_{i}\right) \quad t_{i}=0.001 i \text { and } i \text { is integer-valued } \\
& \frac{\partial R}{\partial P}=R_{P}\left(P, E_{0}, n\right)=1-\frac{E_{0}{ }^{n+1}}{\left(P^{n}+E_{0}\right)^{1 / n}}
\end{aligned}
$$

The equations above are the same as Eq. (6a) and (2a) in the manuscript, respectively. Substituting Eq. (S1), Eq. (S3) becomes a one-variable function of $t$, i.e.,

$$
\frac{\partial R}{\partial P}=R_{P}(t)=1-\frac{(10.3 t+1087)^{(-0.19 t+3)+1}}{\left.\left[(-39.2 t+655)^{n}+(10.3 t+1087)^{(-0.19 t+3)}\right)\right]^{1 /(-0.19 t+3)}}
$$

Then we can calculate $R_{P}\left(t_{i}\right)$ for each $i$ in Eq. (S2) and we thus obtain:

$\Delta R_{P}$ for Subperiod $1=8.58$

(3) Using Eq. (S1) and Eqs. (6b-6c) and (2b-2c) in the manuscript, we can obtain $\Delta R_{E_{0}}$, and $\Delta R_{n}$ in a similar way:

$$
\Delta R_{E_{0}} \text { for Subperiod } 1=-0.95 \text {, and } \Delta R_{n} \text { for Subperiod } 1=5.91
$$

3) Repeating Step 2, we get $\Delta R_{P}, \Delta R_{E_{0}}$, and $\Delta R_{n}$ for each subperiod.

Table S3 Calculated $\Delta R_{P}, \Delta R_{E_{0}}$, and $\Delta R_{n}$ for each subperiod

\begin{tabular}{|l|c|c|c|c|c|c|c|c|c|c|c|}
\hline & $R$ & $P$ & $E_{0}$ & $n$ & $\Delta R$ & $\Delta P$ & $\Delta E_{0}$ & $\Delta n$ & $\Delta R_{P}$ & $\Delta R_{E_{0}}$ & $\Delta R_{n}$ \\
\hline Subperiod 1 & 37.1 & 616 & 1097 & 2.9 & -3.5 & -39.2 & 10.3 & -0.19 & -8.6 & -0.95 & 5.9 \\
\hline Subperiod 2 & 33.1 & 648 & 1094 & 3.2 & -4.0 & 32.4 & -3.5 & 0.38 & 6.6 & 0.3 & -10.7 \\
\hline Subperiod 3 & 25.2 & 624 & 1074 & 3.5 & -7.9 & -24.7 & -19.8 & 0.22 & -4.5 & 1.6 & -4.9 \\
\hline
\end{tabular}

4) Summing up the last three columns in Table S3 respectively, we obtained the same results as in the line 2 in Table 3:

$\Delta R_{P}$ for the Batalling $\mathrm{Ck}=-6.49$,

$\Delta R_{E 0}$ for the Batalling $\mathrm{Ck}=0.95$,

and $\Delta R_{n}$ for the Batalling $\mathrm{Ck}=-9.74$

\section{$R$ codes}

\section{To calculate $n$}

nCal<-function(P,EO, R) \{ 


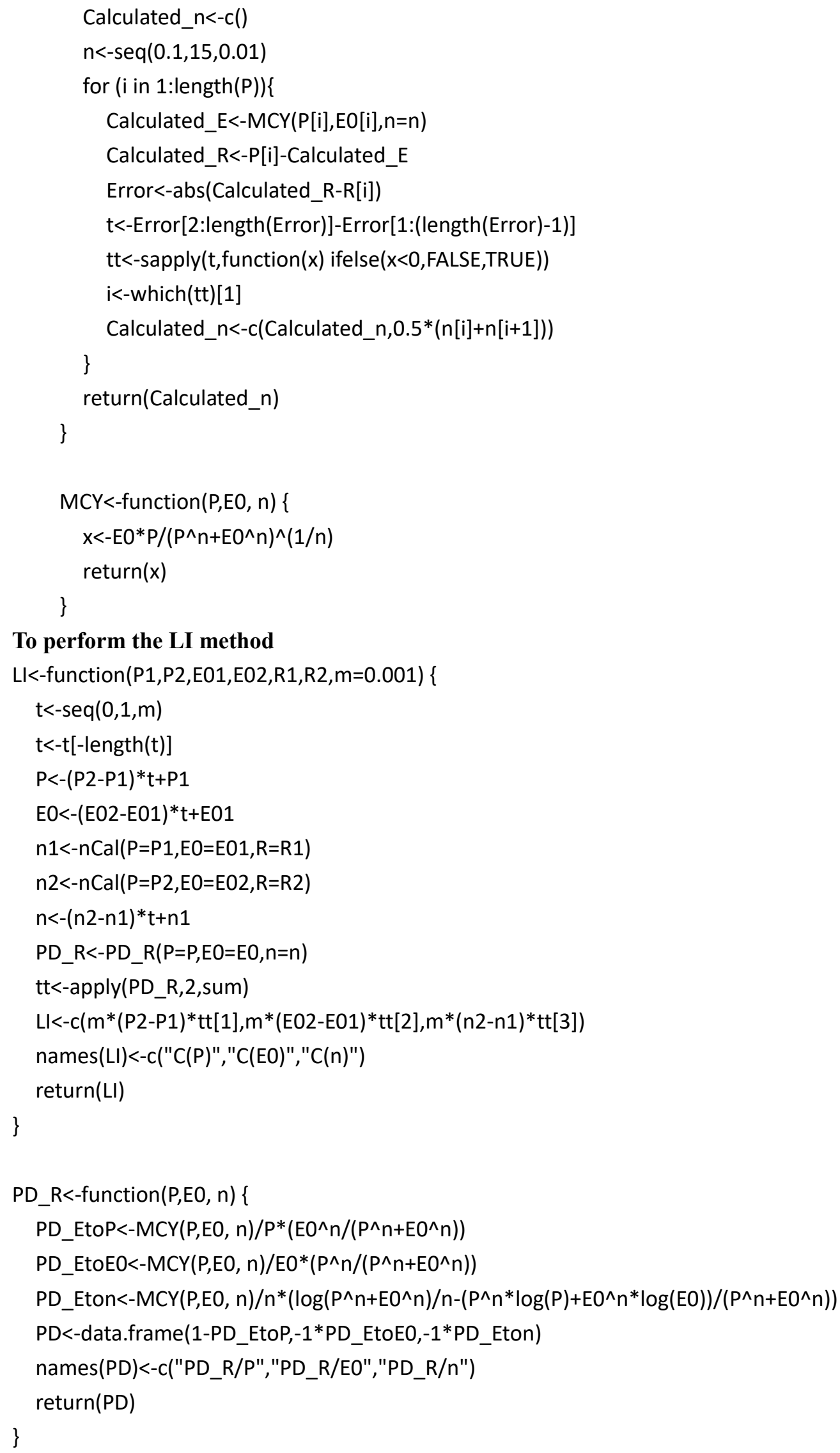


Table S4 Comparisons of the path-averaged sensitivities with the point sensitivities of runoff $a, b$

\begin{tabular}{|r|r|r|r|r|r|r|}
\hline $\begin{array}{l}\text { Catchm- } \\
\text { ent NO. }\end{array}$ & $\overline{\lambda_{P}}$ & $\overline{\lambda_{E 0}}$ & $\overline{\lambda_{n}}$ & $\lambda_{P}$ & $\lambda_{E 0}$ & $\lambda_{n}$ \\
\hline 1 & 0.68 & -0.55 & -17 & 0.621 & -0.39 & -71.8 \\
\hline 2 & 0.2 & -0.08 & -27.3 & 0.227 & -0.1 & -30.9 \\
\hline 3 & 0.58 & -0.36 & -26.7 & 0.68 & -0.42 & -79 \\
\hline 4 & 0.3 & -0.16 & -30.5 & 0.39 & -0.2 & -50.1 \\
\hline 5 & 0.33 & -0.14 & -43.1 & 0.394 & -0.19 & -59.4 \\
\hline 6 & 0.29 & -0.16 & -26.5 & 0.352 & -0.2 & -34.9 \\
\hline 7 & 0.71 & -0.32 & -223 & 0.781 & -0.33 & -299 \\
\hline 8 & 0.49 & -0.26 & -77.9 & 0.478 & -0.27 & -64.9 \\
\hline 9 & 0.16 & -0.07 & -11.8 & 0.161 & -0.07 & -17.6 \\
\hline 10 & 0.72 & -0.45 & -57.3 & 0.74 & -0.53 & -61.1 \\
\hline 11 & 0.25 & -0.15 & -19.8 & 0.29 & -0.17 & -22.5 \\
\hline 12 & 0.34 & -0.18 & -37.2 & 0.393 & -0.21 & -48.6 \\
\hline 13 & 0.68 & -0.22 & -275 & 0.719 & -0.25 & -303 \\
\hline 14 & 0.7 & -0.23 & -326 & 0.745 & -0.24 & -378 \\
\hline 15 & 0.66 & -0.19 & -320 & 0.708 & -0.2 & -378 \\
\hline 16 & 0.65 & -0.19 & -315 & 0.692 & -0.19 & -363 \\
\hline 17 & 0.58 & -0.17 & -153 & 0.602 & -0.17 & -175 \\
\hline 18 & 0.32 & -0.12 & -50.1 & 0.402 & -0.16 & -69.6 \\
\hline 19 & 0.2 & -0.06 & -29.2 & 0.234 & -0.09 & -34 \\
\hline
\end{tabular}

a $\overline{\lambda_{P}}\left(10^{-3} \mathrm{~m} 10^{-3} \mathrm{~m}^{-1}\right), \overline{\lambda_{E 0}}\left(10^{-3} \mathrm{~m} 10^{-3} \mathrm{~m}^{-1}\right)$, and $\overline{\lambda_{n}}$ (dimensionless) represent the pathaveraged sensitivities of runoff to precipitation, potential evaporation, and catchment properties. If the evaluation period comprised only one subperiod, $\overline{\lambda_{P}}, \overline{\lambda_{E 0}}$ and $\overline{\lambda_{n}}$ was calculated as: $\overline{\lambda_{P}}=\Delta R_{P} / \Delta P, \overline{\lambda_{E_{0}}}=\Delta R_{E_{0}} / \Delta E_{0}$, and $\overline{\lambda_{n}}=\Delta R_{n} / \Delta n$. If the evaluation period comprised $\quad N>1 \quad$ subperiods, the equations became: $\overline{\lambda_{P}}=\sum_{i=1}^{N}\left|\Delta R_{P i}\right| / \sum_{i=1}^{N}\left|\Delta P_{i}\right|$, $\overline{\lambda_{E_{0}}}=-\sum_{i=1}^{N}\left|\Delta R_{E 0}\right| / \sum_{i=1}^{N}|\Delta E 0 i|$, and $\overline{\lambda_{n}}=-\sum_{i=1}^{N}\left|\Delta R_{n i}\right| / \sum_{i=1}^{N}\left|\Delta n_{i}\right|$, where the subscript $i$ denotes the $i$ th subperiod.

${ }^{\mathrm{b}} \lambda_{P}, \lambda_{E 0}$, and $\lambda_{n}$ represent the point sensitivities of runoff of the total differential method, which was calculated by substituting the observed mean annual values of the reference period into Eq. (2). 